\title{
Cytokines in the blood and semen of infertile patients
}

\author{
ANNA HAVRYLYUK ${ }^{1}$, VALENTYNA CHOPYAK ${ }^{1}$, YARYNA BOYKO ${ }^{l}$, IRYNA KRIL ${ }^{l}$, \\ MACIEJ KURPISZ ${ }^{2}$
}

${ }^{1}$ Department of Clinical Immunology and Allergology, Danylo Halytsky Lviv National Medical University, Lviv, Ukraine ${ }^{2}$ Department of Reproductive Biology and Stem Cells, Institute of Human Genetics, Polish Academy of Sciences, Poznan, Poland

\begin{abstract}
Cytokines have been important mediators of the immunity and can be involved in numerous processes in the male genital tract including acting as immunomodulatory elements within the male gonad.

The aims of this study were: 1) to detect pro- and anti-inflammatory cytokine levels in the control group and subgroups of infertile men; and 2) to set up the practical recommendations concerning determination of cytokine levels for the male infertility diagnosis.

Observations were performed in a group of 82 men: healthy controls $(n=27)$ and infertile patients $(n=55)$. The male infertility group was further subdivided into patients with: varicocele $(n=22)$, idiopathic infertility $(n=13)$ and partners of couples with recurrent spontaneous abortion $(R S A ; n=20)$. Semen analysis was determined following WHO criteria. The cytokine interleukin $1 \beta(I L-1 \beta), I L-6$, $I L-10, I L-18$; tumor necrosis factor $\alpha(T N F-\alpha)$, interferon $\gamma(I F N-\gamma)$ and transforming growth factor $\beta 1$ (TGF- $\beta 1)$ contents in serum and seminal plasma were determined by quantitative ELISA.

An interesting marker of male infertility appears to be TGF- $\beta 1$ (blood) significantly elevated in idiopathically infertile males and in the RSA group. Besides elevated TGF- $\beta 1$ in a group of idiopathic infertility significantly elevated IL-10, IL-18,IFN- $\gamma$ (blood) and statistically decreased IL-1 $\beta$ while increased IFN- $\gamma$ were revealed in seminal plasma compared to healthy controls.

We may postulate novel cytokine micropatterns for patients with different background of infertility. Therefore, circulating cytokines: IL-1 $\beta, I L-10, I L-18, T G F-\beta 1, I F N-\gamma$ and IL-1 $\beta, I F N-\gamma$ and TGF- $\beta 1$ in seminal plasma should be extended in evaluation of specific types of male infertility.
\end{abstract}

Key words: circulating cytokines, seminal plasma cytokines, male infertility.

(Cent Eur J Immunol 2015; 40 (3): 337-344)

\section{Introduction}

Infertility is defined as inability of couples to achieve pregnancy following one year of unprotected intercourse. By this criterion, infertility affects $13-18 \%$ of couples and male factor accounts for up to half of all the cases [1].

Development of male infertility is influenced by many background diseases and/or risk factors. A substantial increase in the infertility risk is noted mainly in male population, especially when exposed to environmental factors, such as diet and general lifestyle. However, it is important to recognize also genetic variants associated with common complex diseases as male infertility which can be only "one piece of the puzzle" making up an overall risk [2]. No cause can be found using routine diagnostic work-up in $10-15 \%$ of infertile couples. A male contribution to infertility is found to be $45-50 \%$ of the cases. Out of them, in $30-45 \%$, the cause has not been identified (idiopathic male infertility). Besides relevant somatic and genetic factors, male infertility may be caused by existing co-morbidities as general/systemic diseases, malignancies (including lymphomas, leukemias), obesity and others [3]. Testicular dysfunction causing infertility can be multifactorial, particularly in patients with associated chronic illnesses [4-8]. Furthermore, inflammatory diseases or chronic renal failure, immunosuppressive treatment and frequent genetic abnormalities associated with sex chromosomes (Klinefelter's syndrome and Y-chromosomal microdeletions), and also the presence of anti-sperm antibodies (ASA) may influence reproductive functions [9].

One of the main male infertility causes is varicocele which is present in $2-22 \%$ of the adult male population $[10,11]$. Immunological and hormonal factors are vitally important for the observed sperm motility reduction and they appear to have a certain role in varicocele-related infertility [12]. Data on varicocele indicate seminological

Correspondence: Maciej Kurpisz, Department of Reproductive Biology and Stem Cells, Institute of Human Genetics, Polish Academy of Sciences, Strzeszynska 32, 61-049 Poznan, Poland, e-mail: kurpimac@man.poznan.pl 
parameters as contradictory since males with varicocele show infertility with variable spermiograms. Moreover, some males with varicocele appear to be fertile, but their fertility potential might decline gradually [13]. Acute and chronic infections of the genitourinary (GU) tract play also an important role in male factor infertility. They are involved in up to $15 \%$ of male infertility. Although GU tract infections can lead to elevated numbers of seminal leukocytes (SL), there are multiple other reasons for seminal leukocytes presence independent of infectious etiology. A history of autoimmunity can be also potentially noninfectious with an elevated SL content, as the role of seminal leukocytes is mostly to scavenge the faulty sperm [14]. However, in most studies regarding male genital infection/ inflammation, cytokines and their various soluble receptors have been distinctly expressed in the seminal plasma independent of the presence of infections $[15,16]$.

The incidence of infertility among males is still difficult to be precisely established, however current evidence suggests that approximately $20-25 \%$ of young men has poor semen quality and in $30-50 \%$ of couples undergoing in vitro fertilization (IVF), a male factor principally contributes to infertility. Male infertility is not outwardly obvious because macroscopically the ejaculates of fertile and infertile men frequently appear to be similar. It is only when couples fail to achieve conception, that male infertility may be suspected and laboratory tests (e.g. semen analysis) are required to establish this reliably. In recent years, a question whether or not the incidence of male infertility has been really increased is intensely debated [17]. A lot of evidence suggests that somatic testicular cells (Sertoli, Leydig, peritubular cells) in physiological conditions produce vast amounts of cytokines, such as interleukin 1 (IL-1), and IL-6, which take part in spermatogenesis and semen maturation [18-20].

In the male gonad, cytokines are produced physiologically and are involved in its normal function. In consequence, they must also appear as natural components of seminal plasma [21]. A network of cytokines, chemokines, and growth factors, such as interleukins (ILs), IL-1 $\alpha$ and IL-1 $\beta$, IL-2, IL-4 - IL-8, IL-10 - IL-13, IL-17, and IL18, their soluble receptors and antagonists (e.g., IL-1RA, sR IL-2, sR IL-6), TNF- $\alpha$, transforming growth factor (TGF) family of cytokines ( $\alpha$ and $\beta$, and $\gamma$ ), granulocyte colony-stimulating factor (GM-CSF), and macrophage inflammatory proteins $\alpha$ (MIP- $1 \alpha)$ and $\beta$ (MIP- $\beta$ ), were shown to be present in human semen [21, 22].

The precise origin and regulation of the cytokines in the male genital tract are still under investigation. The main sources of cytokines occurring in the male reproductive tract are testes and, among others, testicular macrophages, although some cytokines (IL-1, IL-6) are also produced by integral testicular somatic cells, as are the Leydig and Sertoli cells [18]. They can affect every aspect of reproductive physiology and fertility regulation. Tumor necrosis factor $\alpha$ (TNF- $\alpha$ ), interleukins, IFN- $\gamma$, and some of their soluble receptors present in semen are also secreted by immune cells, mesenchymal cells, Sertoli cells and spermatogonia. Cytokines such as IL-6, IL- 8 and TNF- $\alpha$ may therefore naturally occur in human semen [23].

Testes are considered to be an immunoprivileged organ due to the developed tolerance to sperm autoantigens secreted (leaking) during sexual maturity from male, germ cells. This phenomenon supports development of spermatogenesis. Testes are protected against autoimmune diseases due to immunological/anatomical blood-testis barrier (BTB) which mostly prevents the antigenic leakage out of germ cells to the immune system and transition of antibodies from endothelium to the lumen of seminiferous tubules. Immunosuppressive factors secreted by macrophages, the Sertoli, Leydig, and peritubular cells, and a limited presence of activated T-lymphocytes (particularly of CD8+ phenotype) and regulatory Treg lymphocytes are also in action. Maintaining of the balance between systemic immunity and the "immunoprivileged" status of gonad depends, among others on the function of cytokines which perform both roles as proinflammatory mediators as well as its inhibitors [24].

Blood-testis-barrier regulation is ensured by activated TGF- $\beta$ and TNF- $\alpha$ systems. Receptors for these cytokines are located on Sertoli cells which regulate migration of germ cells through the barrier to the adluminal compartment [18]. Cytokines may influence apical ES (ectoplasmic specialization) as well as the whole BTB altering protein levels present in biological membranes as $\mathrm{N}$-cadherin in the case of apical ES and occluding zones in BTB. A consequence of their action is an increase in protein endocytosis and intracellular endosome-mediated degradation $[18,25]$.

Tumor necrosis factor $\alpha$ function is different from the remaining cytokines and it acts on the androgenic receptor regulating testosterone activity. Therefore, cytokines of the TNF-family promote cell survival during spermatogenesis [26]. Tumor necrosis factor $\alpha$ can also influence spermatogenesis and the sperm function by multiple tasks, such as reducing the production of testosterone, and/or adjusting the proportion of hormones in parts of testis (from Sertoli cells). Besides, TNF- $\alpha$ has a direct effect on sperm motility [23]. Thus, TNF- $\alpha$ has been implicated in steroidogenesis regulation as well as in germ cell survival, and in Sertoli and Leydig cell secretory function.

Cytokines do not only play a role in directing germ cells to apoptosis, but TNF- $\alpha$, TGF- $\beta 2$, and TGF- $\beta 3$ together with testosterone have capacity to regulate spermatogenesis $[27,28]$. For example, TGF- $\beta$ regulates a variety of cellular processes, including the secretory function of Leydig and Sertoli cells, testis biological development and spermatogenesis intensity [29].

As previously stated, human semen contains a variety of different cytokines and other immunological factors but 
their effects on semen quality and sperm function are still the subject of intense debate [30-34].

The aims of the present study were: 1) to detect the levels of pro- and anti-inflammatory cytokines in the control group of healthy men and in different subgroups of infertility; and 2) to emphasize determination of cytokine levels as a possible addition to male infertility diagnosis.

\section{Material and methods}

\section{Patients}

Observations were carried out on 82 male individuals consisting of 27 healthy controls and 55 infertile patients. All these patients $(n=55)$ attended the out-patient for couples infertility and were sampled before the process of medical treatment began. They formed three infertile subgroups to be studied: patients with varicocele $(n=22)$; patients with idiopathic infertility $(n=13)$; and somatic healthy men whose wives had early recurrent spontaneous miscarriages (within the first trimester) $(n=20)$. Serum samples were collected during a routine procedure of drawing venous blood without anticoagulant. Seminal plasma samples were collected from completely liquefied ejaculates and were centrifuged at $300 \mathrm{~g}$ for 20 minutes. Collected males were individually informed on the purpose of the study and agreed to have used their blood and semen samples. A project encompassing this research (National Science Centre of Poland) was approved by the Local Bioethical Committee of Lviv National Medical University which granted permission for patients participation.

\section{Methods}

\section{Semen analysis}

Male subjects were sexually abstinent for 3-5 days. Semen samples were obtained by masturbation. After complete semen liquefaction, volume, appearance, fluidity and basic sperm parameters were determined following the procedures described by the WHO Manual (WHO, 2010). The routine seminological analysis was carried out estimating sperm density, motility, viability and morphology.

\section{The cytokine contents in serum and seminal plasma}

The serum and seminal plasma samples were kept at $-20^{\circ} \mathrm{C}$ and cytokine contents of IL-1 $\beta$, IL-6, IL-10, IL-18; TNF- $\alpha$, IFN- $\gamma$ and TGF- $\beta 1$ were determined using the quantitative ELISA method. The kits detecting IL- $1 \beta$, IL-6, IL-10, IL-18, TNF- $\alpha$ and IFN- $\gamma$ were obtained from VECTOR-BEST (Novosibirsk, Russia); while the kits detecting TGF- $\beta 1$ - from DRG Diagnostics, (Germany). Sensitivity limits for cytokine detection were as follows: for IL- $1 \beta-1 \mathrm{pg} / \mathrm{ml}$, IL-6 - $0.5 \mathrm{pg} / \mathrm{ml}$, IL- $10-1 \mathrm{pg} / \mathrm{ml}$,
IL-18 - $5 \mathrm{pg} / \mathrm{ml}$, TNF- $\alpha-1 \mathrm{pg} / \mathrm{ml}, \mathrm{IFN}-\gamma-5 \mathrm{pg} / \mathrm{ml}$, TGF- $\beta 1-1.9 \mathrm{pg} / \mathrm{ml}$.

\section{Statistics}

Since interleukin levels were normally distributed, mean and standard deviations were calculated. These values as well as probability analyses were calculated using the Mann-Whitney method.

\section{Results}

We have found statistically significant differences in various subgroups of infertile patients in comparison to the control group of healthy males.

Thus, statistically significant elevated levels of TGF- $\beta 1$ were found in infertile males with idiopathic infertility (no conception) (Fig. 1A) and in male partners of couples with early recurrent miscarriages (Fig. 1B) compared to healthy controls.

We have also found statistically significant elevated levels of IL-10 (Fig. 2A), IL-18 (Fig. 2B) and IFN- $\gamma$ (Fig. 2C) in blood serum samples of a group of idiopathically infertile males comparing to the male healthy control group. Interestingly in this group of infertile males, the IL- $1 \beta$ level in seminal plasma was significantly decreased (Fig. 3A) while IFN- $\gamma$ was significantly increased (Fig. 3B).

\section{Discussion}

The local bioactivity of substances released by seminal leukocytes (SL) during the inflammatory reactions in the genitourinary tract and reciprocal interactions among the particular inflammatory mediators (bacteria, leukocytes, proinflammatory cytokines) has been an interesting field, which has been recently often taken up due to its possible clinical implications. Creation of an in vitro model for inflammatory conditions enables observations of particular pro-inflammatory mediators and their influence on the qualitative and quantitative changes at oxygen metabolism in semen, which obviously cannot be traced in a healthy or a sick patient in in situ condition [18].

The high levels of some cytokines present during persistent infection/inflammation in the male genital tract may augment the peroxidation process and affect sperm function with a subsequent development of infertility. Many authors suggest that the measurement of IL-6, IL-8, and/ or IL-18 contents in seminal plasma can be a sensitive marker of early infection/inflammation in the male genitourinary tract and a signal for fast intervention with an anti-inflammatory treatment [35-38]. The role and function of leukocytes in semen, as well as their clinically relevant pathological threshold values, are a matter of debate [39]. The IL-1 $\beta$, IL-4 and IL-10 contents in seminal plasma are closely related to male reproductive ability. The increase 
A

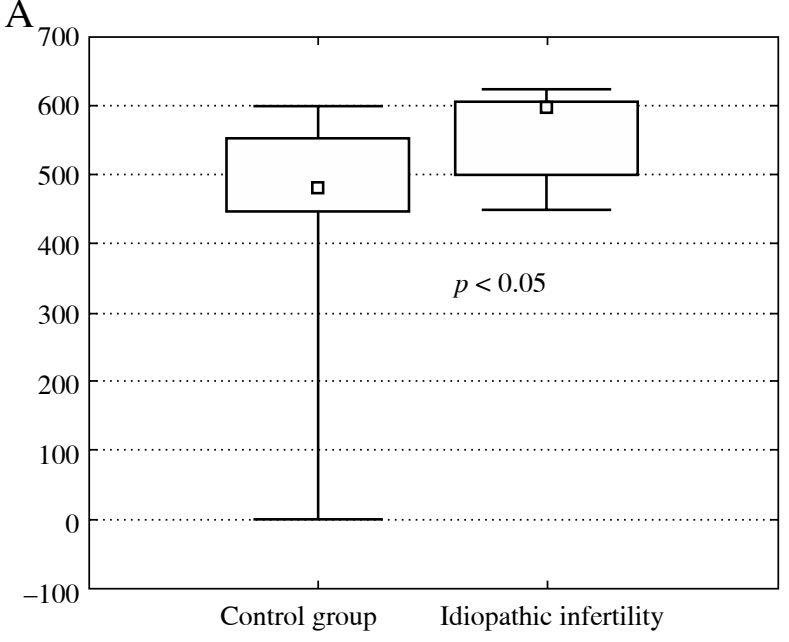

$\mathrm{B}$

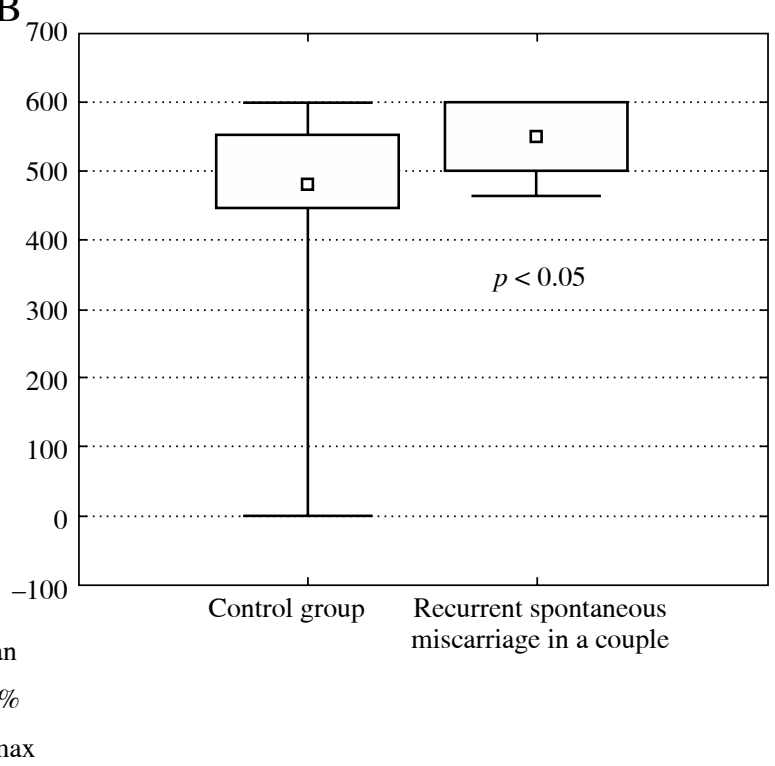

Fig. 1A-B. The TGF- $\beta 1$ levels in blood samples of the control group of healthy males and a group of infertile individuals: with A) idiopathic infertility (no conception), B) partners in couples with recurrent spontaneous abortions

or decrease in these cytokines reflect the status of immunity and/or infection of the reproductive system, and influence sperm function [40,41]. A lot of studies dedicated to the effects of cytokines and growth factors on sperm function provided, however, controversial results. For example, most investigators suggested that the cytokines detected in seminal plasma are associated with the leukocytospermia but these are not frequently the cause of semen abnormalities [see, for review, 18].

In the male genital system, some cytokines (IL-1 and IL-6) can be produced and secreted not only by somatic cells, but also by germ cells. It is possible therefore that cytokines may act not only towards somatic cells, but also towards the germ cells both in an autocrine and paracrine fashion. It is possible that cytokines may act during spermatogenesis, sperm maturation, sperm transport, and even during the fertilization process itself. Finally, cytokines can be released by various immunocompetent cells present in the male genital tract, which can be the major source of these factors produced in response to foreign antigens, pathogens, and also in inflammatory conditions [18]. It was demonstrated that antisperm antibodies can be a common reason of male infertility also in association with the cytokine presence and that infection/inflammation of the male genital tract, systemic infection or illness may deteriorate the testicular function [24]. Conversely, secondary autoimmune orchitis can be represented by testicular vasculitis associated with systemic autoimmune diseases such as polyarteritis nodosa, Behçet's disease, and rheumatoid arthritis [42, 43].
Generally, effects of different cytokines on the semen quality and sperm function can be very different. Current literature provides the following issues: 1) there exists a controversy concerning the role of particular cytokines in the fertilization process; 2) a lot of studies indicate the lack of any connection between the cytokine levels and semen quality or infertility status; 3 ) a number of studies, however, point out to the observed relationship between the cytokine hypersecretion and the deterioration of semen parameters or sperm fertilization ability; 4 ) some authors have not found similar good effects of TNF- $\alpha$ and IL-8 towards sperm motility and the acrosome reaction; 5) IFN- $\gamma$ seems to show an ability to inhibit sperm motility; 6) IL-12 plays a certain role in inducing of male infertility; 7) there has been observed a strong relationship of increased seminal plasma TNF- $\alpha$ levels with sperm DNA/chromatin damage and decreased (progressive and total) sperm motility; 8) the IL-6 levels found in semen of immune infertile patients was significantly higher and revealed a clear negative correlation with the amount of sperm [23, 28, 38, 44-47].

The positive effects of cytokines in the male reproductive function can be summarized as follows: 1 ) multifactorial interactions are attributed to IL-18 regarding homoeostasis of cell proliferation and apoptosis in the testis; 2 ) the proinflammatory cytokines are paracrine defense factors in the male gonad; 3) the anti-inflammatory cytokines are the soluble immunosuppressive factors, which protect testis against autoimmune reaction in collaboration with anatomical blood-testis-barrier (BTB) [24, 48].

It appears that participation of some cytokines in the regulation of fertility may be dependent upon their con- 

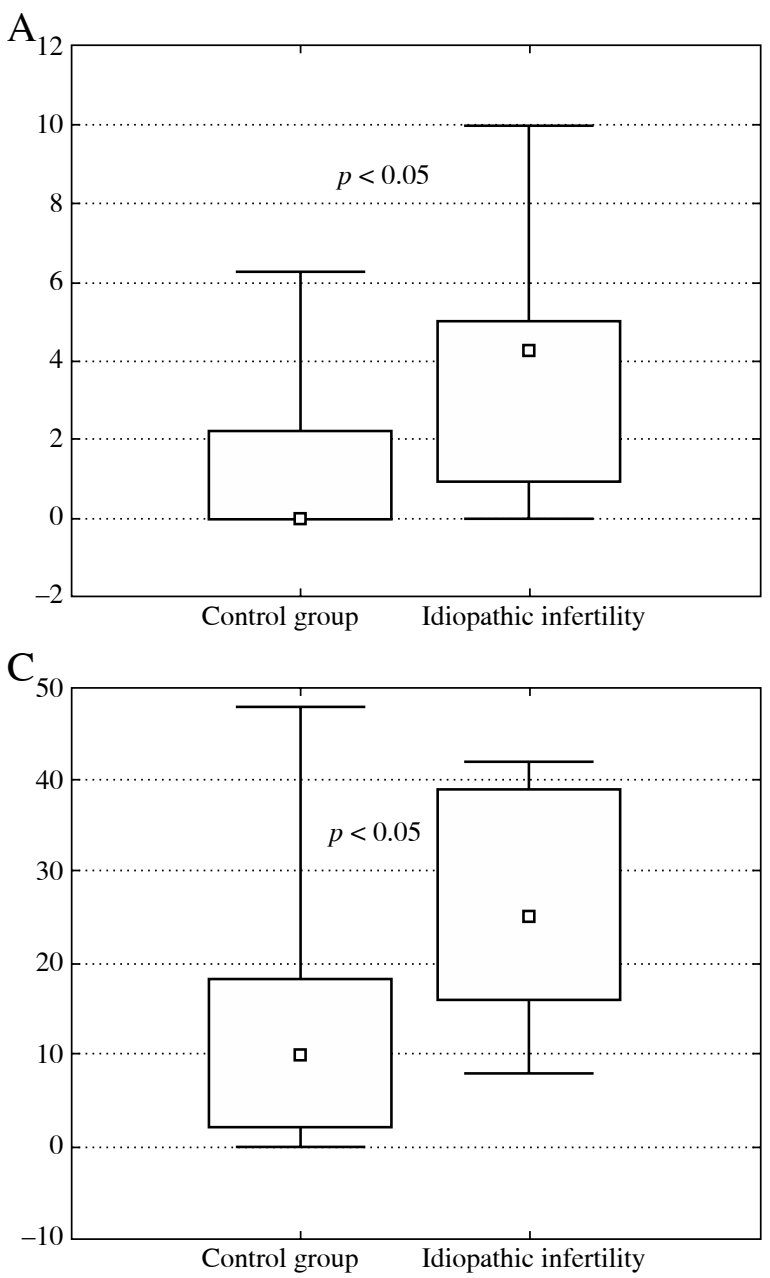

centrations. High levels of certain cytokines in semen may correspond to the quality of semen parameters, such as sperm count, motility and morphology, or sperm-oocyte penetration rates.

An interesting observation arising from our studies is a potentially significant role of TGF- $\beta 1$ in different forms of male infertility (Fig. 1). Its elevated levels were found in: a) a group of idiopathically infertile individuals, and b) male partners in couples with recurrent spontaneous abortion. Transforming Growth Factor $\beta$ in normal range regulates a variety of cellular processes, including the secretory function of Leydig and Sertoli cells, testis development and spermatogenesis. This cytokine (in high concentration) in concert with TNF- $\alpha$ probably plays also a role in directing germ cells to apoptosis [27, 28]. Excessive Transforming Growth Factor $\beta 1$ signaling is associated with pathological scarring and fibrotic diseases. TGF- $\beta 1$ activation and signaling have multiple points of regulation that modify its transcriptional and functional immunoregulatory effects. For example, in Crohn's disease dominant inhibitory Smad-7-receptor blocks TGF- $\beta 1$ immunoregu-

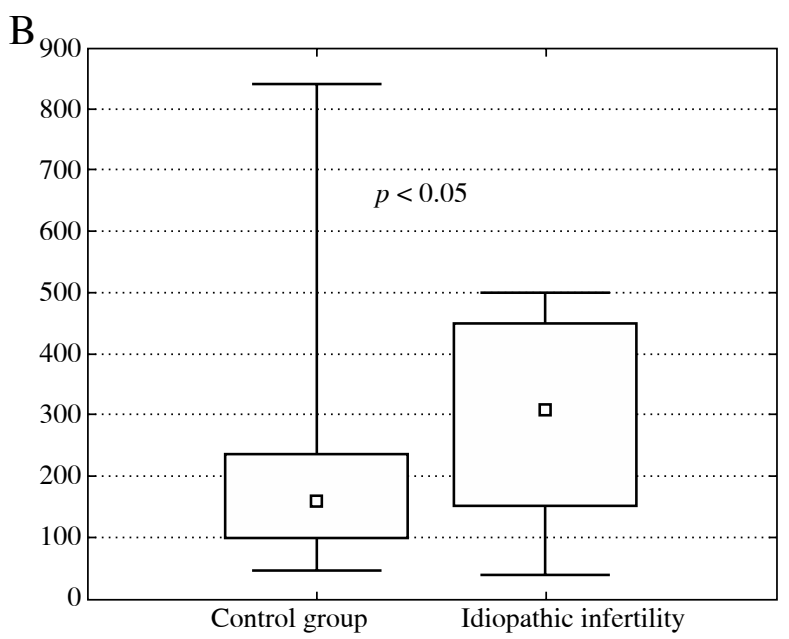

$$
\begin{aligned}
& \square \text { Median } \\
& \square 25-75 \% \\
& \text { 工 Min-max }
\end{aligned}
$$

Fig. 2A-C. Cytokine levels in blood samples of the control group of healthy males and a group of idiopathically infertile individuals (no conception): A) IL-10, B) IL-18, C) IFN- $\gamma$

latory actions permitting T-cell proinflammatory signaling. The cytokines of the TGF- $\beta$ family play a critical role in the regulation of cell cycle progression and differentiation, in both normal and pathological conditions [29].

Since TGF- $\beta 1$ was found consequently to be elevated in the blood samples of two male infertility subgroups (comparing to control healthy males), this undoubtedly makes its biological meaning for male infertility and so far has no direct reflection in reported studies on cytokines and its relationship with male reproduction. Prospective studies should follow this direction in near future.

Striking data were found in biological fluids (both in blood and seminal plasma) of patients with idiopathic infertility (no conception). Generally, there is no good literature documentation of the studies of immune parameters in this male population. We have found statistically increased levels of IL-10, IL-18, TGF- $\beta 1$ and IFN- $\gamma$ in blood samples; while an increased level of IFN- $\gamma$ and decreased level of IL-1 $\beta$ in seminal plasma $(p<0.05)$ in this group comparing to healthy males (Figs. 2 and 3 ). This group of patients should be better examined in fu- 

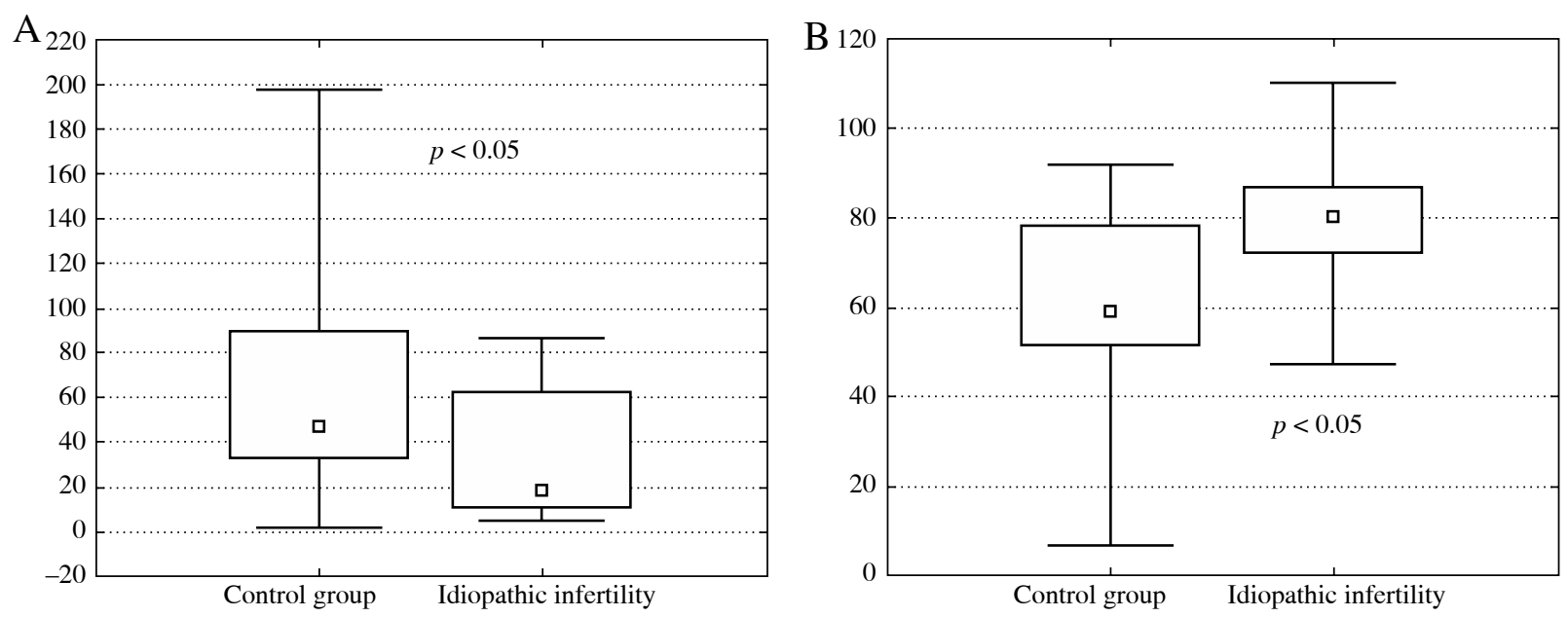

\section{Median \\ $\square 25-75 \%$}

\section{工 Min-max}

Fig. 3A, B. The cytokine levels in seminal plasma of the control group of healthy males and a group of idiopathically infertile (no conception) individuals: A) IL-1 $\beta$, B) IFN- $\gamma$

ture with regard to the immune causes of infertility development. Specifically since idiopathic infertility constitutes a serious problem to its detection, diagnosis and treatment.

Increased levels of IL-18 can be detected in biological fluids and organs of individuals in several autoimmune animal models. Identification of the causes of uncontrolled IL-18 production and its activity in autoimmune reactions would allow to find novel therapeutic targets to effectively block autoimmune activation and to inhibit concomitant tissue damage [24]. Interleukin 18 increased profile has been detected in our idiopathically infertile group (Fig. 2).

Interferon $\gamma$ seemed to show an ability to inhibit sperm motility as the only cytokine, but this phenomenon was only observed when high concentrations of this cytokine were applied. Thus, it appears that participation of some cytokines in the regulation of fertility may be dependent upon their concentrations [47]. We should not miss the fact that cytokines act in a pleiotropic way and often their interaction may result in an unknown manner.

Numerous reports appeared in which the limited value of standard semen analysis was underlined when assessing male fertilizing potential. Measurement of cytokine concentrations is still not a routine practice. Controlled prospective studies, including a large number of patients, and a wide range of analyzed semen parameters together with patient characteristics and reproductive outcome, are urgently required to answer the question about the future of the novel biomarkers, such as cytokines both in blood and when secreted to seminal plasma.
Several aspects of cytokine research within the male reproductive system indicated: a) the presence of variety of cytokines in human seminal plasma, b) differences in cytokine concentrations between fertile and infertile men, c) negative correlations between some cytokine levels and semen parameters, d) correlations between cytokine levels and leukocyte count as well as, e) usefulness of some cytokines as clinical markers of male infertility subtypes [18].

Generally, we have demonstrated significant changes in cytokines micropattern concerning different cohorts of infertile patients, that is patients with idiopathic infertility, and in male partners of couples with early miscarriages. Among those, a group of infertile males with idiopathic background looks much more intriguing than the remaining one according to the range of cytokines significantly engaged in its etiology. Probably this group of patients should be further investigated for immunological reasons for infertility development. Prospectively, we may suggest extending the studies of immunological parameters addressing the issue whether these factors may profile male infertility with specific cytokine micropattern for each infertility disorder allowing them to be better differentiated and more precisely diagnosed (and treated) using more appropriate means.

\section{Conclusions}

We have demonstrated significant changes in immunological parameters studied in subgroups of infertile patients with idiopathic infertility, and male partners of couples with early spontaneous miscarriages. 
On the basis of the results obtained in the mentioned male infertility subgroups, we propose extending infertility diagnosis by detecting IL- $1 \beta$, IL- 10 , IL-18, TGF- $\beta 1$, IFN- $\gamma$ in blood serum and IL- $1 \beta$ and IFN- $\gamma$ in seminal plasma in order to clarify whether these factors may constitute a novel approach to the clinical work-up of male infertility management.

The study was supported by the National Science Centre, Poland, grant no. 2011/01/B/NZ2/04836.

The authors declare no conflict of interest.

\section{References}

1. Iammarone E, Balet R, Lower AM, et al. (2003): Male infertility. Best Pract Res Clin Obstet Gynaecol 17: 211-229.

2. Ji G, Long Y, Zhou Y, et al. (2012): Common variants in mismatch repair genes associated with increased risk of sperm DNA damage and male infertility. BMC Med 10: 49.

3. Jungwirth A, Giwercman A, Tournaye H, et al. (2012): European Association of Urology guidelines on Male Infertility: the 2012 update. Eur Urol 62: 324-332.

4. Moraes AJ, Bonfa E, Cocuzza M, et al. (2010): Gonad evaluation in male dermatomyositis. A pilot study. Clin Exp Rheumatol 28: 441-442.

5. Moraes AJ, Pereira RM, Cocuzza M, et al. (2008): Minor sperm abnormalities in young male postpubertal patients with juvenile dermatomyositis. Braz J Med Biol Res 41: 11421147.

6. Silva CA, Bonfa E, Ostensen M (2010): Maintance of fertility in patients with rheumatic diseases needing anti-inflammatory and immunosupressive drugs. Arthritis Care Res (Hoboken) 62: 1682-1690.

7. Silva CA, Hallak J, Pasqualotto FF, et al. (2002): Gonadal function in male adolescents and young males with juvenile onset systemic lupus erytematosus. J Rheumatol 29: 20002005.

8. Suchiro RM, Borba EF, Bonfa E, et al. (2008): Testicular Sertoli cell function in male systemic lupus erythematosus. Reumatology (Oxford) 47: 1692-1697.

9. Silva CA, Cocuzza M, Borba EF, Bonfa E (2012): Cutting-Edge Issues in Autoimmune Orchitis. Clinic Rev Allerg Immunol 42: 256-263.

10. Al-Daghistani HI, Hamad AWR, Abdel-Dayem M, et al. (2010): Evaluation of serum testosterone, progesterone, seminal antisperm antibody, and fructose levels among Jordanian males with a history of infertility. Biochem Res Int 2010: 409640.

11. Kursh ED (1987): What is the incidence of varicocele in a fertile population? Fertil Steril 48: 511.

12. Ficarra V, Porcaro AB, Righetti R, et al. (2002): Antegrade scrotal sclerotherapy in the treatment of varicocele: a prospective study. BJU Int 89: 264-268.

13. Pasqualotto FF, Sundaram A, Sharma RK, et al. (2008): Semen quality and oxidative stress scores in fertile and infertile patients with varicocoele. Fertil Steril 89: 602-607.

14.Domes T, Kirk SLo, Grober ED, et al. (2012): The incidence and effect of bacteriospermia and elevated seminal leukocytes on semen parameters. Fertil Steril 97: 1050-1055.
15. Kokab A, Akhondi MM, Sadeghi MR, et al. (2010): Raised inflammatory markers in semen from men with asymptomatic chlamydial infection. J Androl 31: 114-120.

16. Martinez-Prado E, Camejo Bermúdez MI (2010): Expression of IL-6, IL-8, TNF-alpha, IL-10, HSP-60, anti-HSP-60 antibodies, and anti-sperm antibodies, in semen of men with leukocytes and/or bacteria. Am J Reprod Immunol 63: 233-243.

17.Pacey AA (2012): Assessment of male factor. Best Pract Res Clin Obstet Gynaecol 26: 739-746.

18. Fraczek M, Czernikiewicz A, Kurpisz M (2012): Cytokines and Oxidative Stress in the Germ Line. Chapter 9 in A. Agarwal et al. (eds). Studies on Men's Health and fertility, Oxidative Stress in Applied Basic research and Clinical Practice. DOI 10.1007/978-1-61779-776-7-9, Springer Science + Business Media, LLC 2012; 179-205.

19. Hedger M, Klug J, Fröhlich S, et al. (2005): Regulatory cytokine expression and interstitial fluid formation in the normal and inflamed rat testis are under Leydig cell control. J Androl 26: 379-386.

20. O'Bryan MK, Hedger MP (2008): Inflammatory networks in the control of spermatogenesis: chronic inflammation in an immunologically privileged tissue? Adv Exp Med Biol 636: 92-114.

21. Maegawa M, Kamada M, Irahara M, et al. (2002): A repertoire of cytokines in human seminal plasma. J Reprod Immunol 54: 33-42.

22. Politch JA, Tucker L, Bowman FP, Anderson DJ (2007): Concentrations and significance of cytokines and other immunologic factors in semen of healthy fertile men. Hum Reprod 22: 2928-2035.

23. Qian L, Sun G, Zhou B, et al. (2011): Study on the Relationship Between Different Cytokines in the Semen of Infertility Patients. Am J of Reprod Immunol 66: 157-161.

24.Jacobo P, Guazzone VA, Theas MS, Lustig L (2011): Testicular autoimmunity. Autoimmun Rev 10: 201-204.

25.Xia W, Mruk DD, Lee WM, Cheng CY (2005): Cytokines and junction restructuring duringspermatogenesis - a lesson to learn from the testis. Cytokine Growth Factor Rev 16: 469493.

26. Cavalcanti MC, Steilmann C, Falling K, et al. (2011): Apoptotic gene expression in potentially fertile and subfertile men. Mol Hum Reprod 17: 415-420.

27. Cheng CY, Mruk DD (2010): A local autocrine axis in the testes that regulates spermatogenesis. Nat Rev Endocrinol 6: 380-395.

28.Li MW, Mruk DD, Lee WM, Cheng CY (2009): Cytokines and the junction restructuring events during spermatogenesis in the estis: an emerging new concept of regulation. Cytokine Growth Factor Rev 20: 329-238.

29. Gonzalez CR, Matzkin ME, Frungieri MB, et al. (2010): Expression of the TGF-beta1 system in human testicular pathologies. Reprod Biol Endocrinol 8: 148.

30. Camejo MI, Segnini A, Proverbio F (2001): Interleukin-6 (IL-6) in seminal plasma of infertile men, and lipid peroxidation of their sperm. Arch Androl 47: 97-101.

31.Eggert-Kruse W, Kiefer I, Beck C, et al. (2007): Role for tumor necrosis factor alpha (TNF-alpha) and interleukin 1-beta (IL-1beta) determination in seminal plasma during infertility investigation. Fertil Steril 87: 810-823.

32. Jiwakanon J, Berg M, Persson E, et al. (2010): Cytokine expression in the gilt oviduct: effects of seminal plasma, spermatozoa and extender after insemination. Anim Reprod Sci 119: 244-257. 
33. O'Leary S, Armstrong DT, Robertson SA (2011): Transforming growth factor- $\beta$ (TGF- $\beta$ ) in porcine seminal plasma. Reprod Fertil Dev 23: 748-758.

34. Vera O, Vasqucz LA, Munos MG (2003): Semen quality and presence of cytokines in seminal fluid of bull ejaculates. Theriogenology 60: 553-558.

35.Eggert-Kruse W, Boit R, Rohr G, et al. (2001): Relationship of seminal plasma interleukin (IL)-8 and IL-6 with semen quality. Hum Reprod 16: 517-528.

36. Furuya Y, Akashi T, Fuse H (2003) Soluble Fas and interleukin-6 and interleukin-8 levels in seminal plasma of infertile men. Arch Androl 49: 449-452.

37. Matalliotakis IM, Cakmak H, Fragouli Y, et al. (2006): Increased IL-18 levels in seminal plasma of infertile man with genital tract infections. Am J Reprod Immunol 55: 428-433.

38. Sanocka D, Jedrzejczak P, Szumała-Kaekol A, et al. (2003): Male genital tract inflammation: the role of selected interleukins in regulation of pro-oxidant and antioxidant enzymatic substances in seminal plasma. J Androl 24: 448-455.

39.Lotti F, Corona G, Mancini M, et al. (2011): Ultrasonographic and clinical correlates of seminal plasma interleukin-8 levels in patients attending an andrology clinic for infertility. Int J Androl 34: 600-613.

40.Li H, Shang X, Huang Y (2004): The effects of interleukin-10 and -8 in chronic prostatitis. Zhonghua Nan Ke Xue 10: 486$487,490$.

41.Zhang J, Gao J (2004): Determination of IL-1 beta, IL-4 and IL-10 contents in the seminal plasma of infertile patients and its clinical value. Zhonghua Nan Ke Xue 10: 851-854.

42.Carp HJA, Selmi C, Shoenfeld Y (2012): The autoimmune bases of infertility and pregnancy loss. J Autoimmun 38: j266-j274.

43. Terayama H, Naito M, Qu N, et al. (2011): Intratesticular expression of mRNAs of both interferon $\gamma$ and tumor necrosis factor $\alpha$ is significantly increased in experimental autoimmune orchitis in mice. J Reprod Dev 57: 296-302.

44. Bezold G, Politch JA, Kiviat NB, et al. (2007): Prevalence of sexually transmissible pathogens in semen from asymptomatic male infertility patients with and without leukocytospermia. Fertil Steril 87: 1087-1097.

45. Kopa Z, Wenzel J, Papp GK, Haidl G (2005): Role of granulocyte elastase and interleukin- 6 in the diagnosis of male genital tract inflammation. Andrologia 37: 188-194.

46.Lazaros LA, Xita NV, Chatzikyriakidou AL, et al. (2012): Association of TNF $\alpha$, TNFR1 and TNFR2 polymorphisms with sperm concentration and motility. J Andrology 33: 74-80.

47. Naz RK, Evans L (1998): Presence and modulation of interleukin-12 in seminal plasma of fertile and infertile men. J Androl 19: 302-307.

48. Komsky A, Huleihel M, Ganaiem M, et al. (2012): Presence of IL-18 in testicular tissue of fertile and infertile men. Andrologia 44: 1-8. 